\title{
A Reverse Engineering Approach for Imaging Neuronal Architecture - Large-Area, High-Resolution SEM Imaging
}

\section{Christine A. Brantner, ${ }^{1 *}$ Martin Rasche,${ }^{4}$ Kevin E. Burcham,${ }^{3}$ Joseph Klingfus,${ }^{3}$ Joel Fridmann, ${ }^{3}$} Jason E. Sanabia, ${ }^{3}$ Can E. Korman, ${ }^{2}$ and Anastas Popratiloff ${ }^{1}$

${ }^{1}$ GW Nanofabrication and Imaging Center, The George Washington University, 800 22 ${ }^{\text {nd }}$ St. NW, Washington, D.C. 20052

${ }^{2}$ Department of Electrical and Computer Engineering, The George Washington University, $80022^{\text {nd }}$ St. NW, Washington, D.C. 20052

${ }^{3}$ Raith International Applications Center, Raith America, Inc., 300 Jordan Rd., Troy, NY 12180

${ }^{4}$ Raith GMBH, Konrad-Adenauer-Allee 8, 44263, Dortmund, Germany

*chrisbrantner@email.gwu.edu

Abstract: A reverse engineering approach to scanning electron microscope (SEM) imaging of neuronal architecture provides largearea maps of a sample, which links the function of a cell with its location in a tissue. The Chipscanner's laser interferometer stage and field-of-view mapping allow high-resolution SEM images to be stitched together. This workflow produces accurate, high-resolution maps of tissue over biologically relevant length scales in reasonable time frames. A $2.5 \times 1.8 \mathrm{~mm}$ mouse spinal cord resin section was imaged in less than 24 hours. This is the most accurate, large-area map of neuronal tissue directly acquired by an SEM.

\section{Introduction}

An inherent limitation of traditional high-resolution electron microscopy (EM) approaches is the limited fieldof-view (FOV), which makes it difficult to relate an object of interest to the overall context of the sample in serial block-face scanning electron microscopy (SEM) [1,2] and automated tape-collecting ultramicrotome SEM $[1,3]$. Determining such relationships is pivotal for structure-function relationship assignments in the field of neuroscience. Large-area EM image registrations with seamless junctions between individual images are important in biology to visualize the entire cross section of a tissue or organ.

Spinal cord imaging. The architecture of a spinal cord is complex, and an examination of its cellular structures can only be understood by relating them to the larger cyto-architectural map. The specific location of a neuron in the tissue organization gives valuable information of its function. Thus low-magnification images as well as high-magnification images are required for understanding. EM is the "gold standard" for resolution and contrast in samples, yet the FOV and area that can be imaged is relatively small compared to the area of the organized tissue. This small area of a sample that can be imaged in the EM often does not contain landmarks for "knowing" the location of a cell in relation to the entirety of the tissue or organ.

In this article a reverse engineering approach for imaging a spinal cord is proposed: the Chipscanner (Raith $\mathrm{GmbH}$ ) achieves high-accuracy stitching of adjacent high-resolution SEM images covering large areas of tissue. The combination of high-resolution SEM imaging, laser interferometer stage positioning, and FOV mapping used in this study, produced the most accurate large-area, high-resolution map of spinal cord tissue directly acquired by an SEM instrument reported to date. The use of a reverse engineering approach to the problem of mapping neurons and their subcellular domains could lead to a better understanding of neuronal architecture in the present context of the BRAIN Initiative in the USA (https://www. whitehouse.gov/share/brain-initiative) and the Human Brain Project in Europe (https://www.humanbrainproject.eu).

Microelectronics. With proven accuracy for $22 \mathrm{~nm}$ node technologies, the Chipscanner's high-resolution SEM image

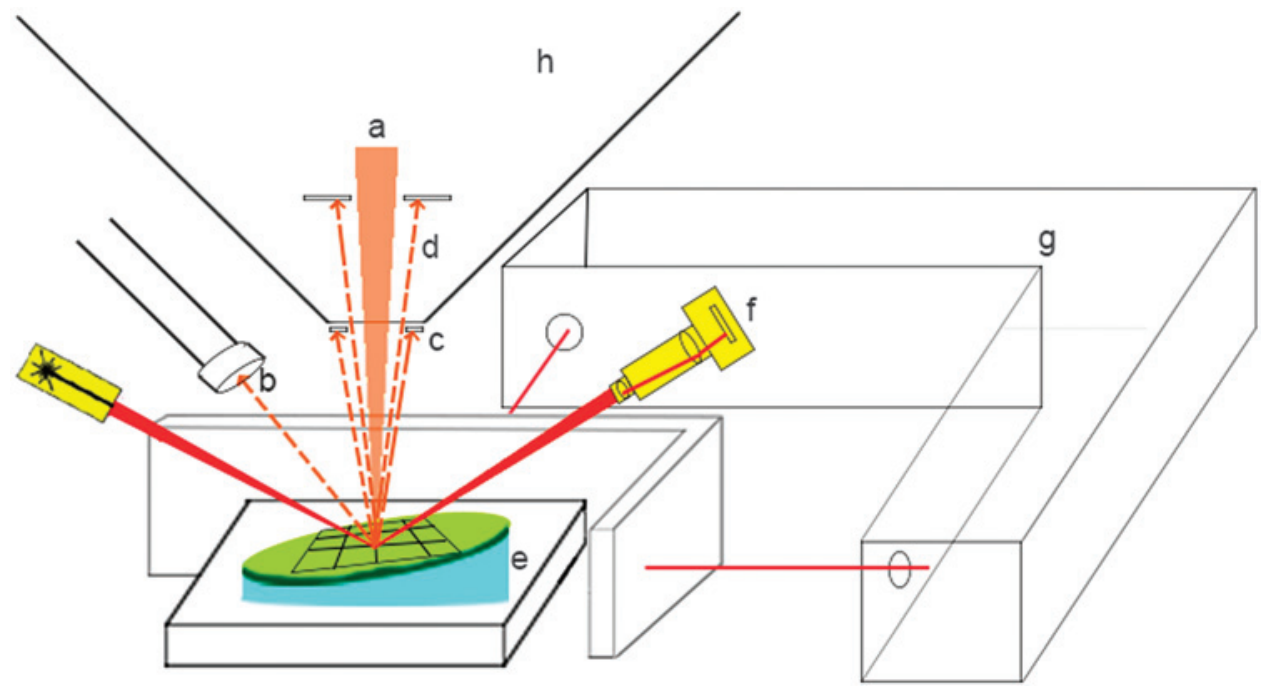

Figure 1: Schematic diagram of the Chipscanner chamber. As with single-beam SEM, the Chipscanner offers a variety of electron detectors and multiple-beam energies. (a) Primary electron beam. (b) EverhartThornley SE detector. (c) Post-lens angular selective backscatter electron detector (AsB). (d) In-lens detector for secondary and/or energy-selective backscatter electrons (EsB). (e) Sample with varying height. (f) Heightsensing apparatus that uses a laser beam to measure the sample height, keeping the sample in focus over large areas. (g) Laser interferometer stage that provides precise sample motion down to single nanometers. (h) Final polepiece of the SEM. 


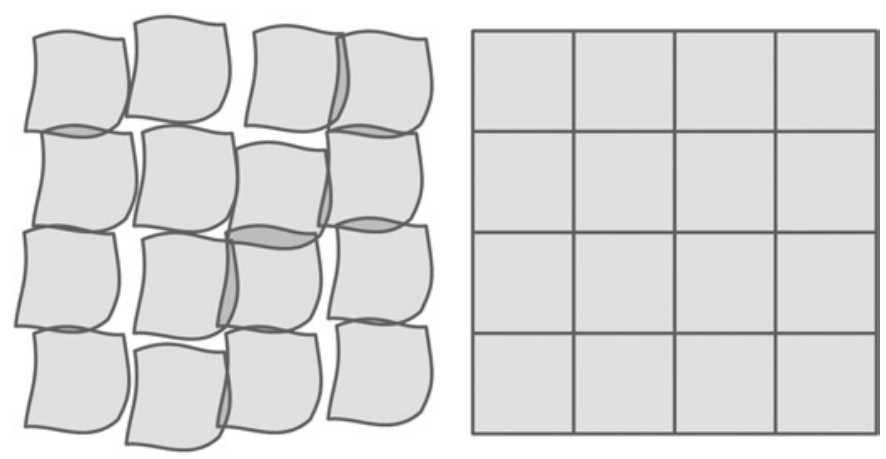

Figure 2: Schematic diagram of tiled images in a large-area mosaic. Left mosaic is without, and right mosaic is with, the laser interferometer stage and FOV corrections that minimize the need for image overlap and software for stitching error removal. Thus, high-resolution SEM images may be collected over large areas.

mosaics have played an essential role in reverse engineering of integrated circuits over the last 15 years [4]. For example, the Chipscanner has been used to reconstruct a recent PC-CPU with elements as small as $22 \mathrm{~nm}$. Using a toolset of de-processing equipment, the CPU was deconstructed layer by layer. Between each de-processing step, the Chipscanner collected large-area, high-resolution SEM images of the exposed layer. The resulting 3D image data were converted to a computer-aided design (CAD) for subsequent feature analysis. Ultimately, the CPU's schematic was extracted with the aim of detecting infringements on intellectual property.

Speed and accuracy. In SEM of large areas and volumes, speed and accuracy are significant factors. Although advances have been made over the years for enhancing SEM image acquisition speed, the Chipscanner is a single-beam system and will not achieve the data collection rates of a multiplebeam SEM [5]. On the other hand, the Chipscanner retains the flexibilities in primary beam landing energy and electron detection schemes that are inherent to single-beam SEMs, and it does not place significant constraints on sample staining procedures required to generate high-contrast SEM images. Furthermore, the Chipscanner brings the advantage of accuracy inherent in electron beam lithography (EBL) instruments.

Stitching errors. In general, SEM imaging over large areas and image stacking in the $Z$ dimension has stitching errors in four dimensions: $X, Y, Z$, and $I$ (signal intensity). The signal intensity, $I$, can vary from image to image because of charging and contamination. Instruments without laser interferometry generally have stitching errors on the order of $1 \mu \mathrm{m}$. Electron beam damage in biological samples can also hamper the matching of adjacent images. Software corrections have not achieved the accuracies necessary to follow synaptic vesicles or microtubules (approx. $20 \mathrm{~nm}$ ) through large areas and volumes of neuronal tissue.

There are two main limitations to software correction of stitching errors. First, only small volumes $<1 \mathrm{~TB}$ can be aligned on a powerful desktop computer [3], whereas a $0.5 \mathrm{~mm} \times 0.5 \mathrm{~mm} \times 0.5 \mathrm{~mm}$ volume at $10 \mathrm{~nm}$ voxel size corresponds to 100 TB. More importantly, such image processing can introduce additional errors into the images via the stretching, shrinking, and twisting of images. Thus the software approach has so far been insufficient for reverse engineering of integrated circuits down to the $22 \mathrm{~nm}$ node.
The positioning accuracy of individual image tiles is critical for the generation of reliable large-area, large-volume SEM image sets.

In this article the Chipscanner's backscattered electron detector was employed to produce an SEM mosaic of a cross section of mouse spinal cord in epoxy resin to demonstrate imaging with high contrast, high resolution, and high dimensional accuracy. This is the first application of a reverse engineering SEM approach to the imaging of neuronal architecture, and the results reported here show promise for delivering the most accurate, high-resolution maps of neuronal tissue over relevant length scales and in reasonable time frames.

\section{Materials and Methods}

The Chipscanner is essentially an SEM on an EBL platform. As such it has a laser interferometer stage and FOV mapping that allow high-resolution SEM images to be stitched and stacked together with high accuracy. As with many commercially available single-beam SEMs, the Chipscanner has a wide range of electron detectors and primary beam energies for optimizing imaging conditions. Furthermore, the Chipscanner lacks a detector array, and so it does not exhibit variation in intensity, $I$, over FOVs of $100 \mu \mathrm{m}$. The Chipscanner produces inherently accurate image data, minimizing or eliminating the need for software corrections of stitching errors.

EBL technologies. Image acquisition in the SEM has instrumental challenges to be overcome. The software on many SEM instruments has the capability to collect images in an array and tile them together. But there are difficulties in such operations, such as a limited FOV size, large numbers of images needed to cover areas of interest, long collection times, stage movement errors and drift, beam position errors and drift, and beam current drift. The Chipscanner (Figure 1) surmounts these difficulties with its built-in EBL technologies, most importantly the laser interferometer stage, the pattern generator, and the lithography-oriented software, all of which are designed around precision. The laser interferometer stage can measure sample position down to single nanometers and below and thus provides accurate and repeatable sample motion. This is used to calibrate the SEM deflection field, ultimately allowing for precise alignment of the adjacent FOVs without the need to stitch images during post-processing (Figure 2). In essence, the laser interferometer acts as the absolute length scale for FOV calibration. After the Chipscanner performs its FOV calibrations, the parameters are stored in the pattern generator. The pattern generator is responsible for beam deflection and signal recording; it automatically applies the FOV calibrations as the electron beam is deflected. The pattern generator collects, stores, and transfers FOV-corrected images, each image having a resolution up to 50,000 pixels by 50,000 pixels. Thus the absolute position of each pixel related to the total area to be reconstructed is known ultimately to the accuracy afforded by the laser interferometer stage. The stitching of $100 \mu \mathrm{m}$ FOVs has a typical accuracy below $10 \mathrm{~nm}$ (mean of measured stitching errors) and a typical precision below $30 \mathrm{~nm}$ (three times the standard deviation of measured stitching errors), in the absence of sample damage from the electron beam. Thus, in most cases the final mosaic does not require any further processing for stitching error removal. 

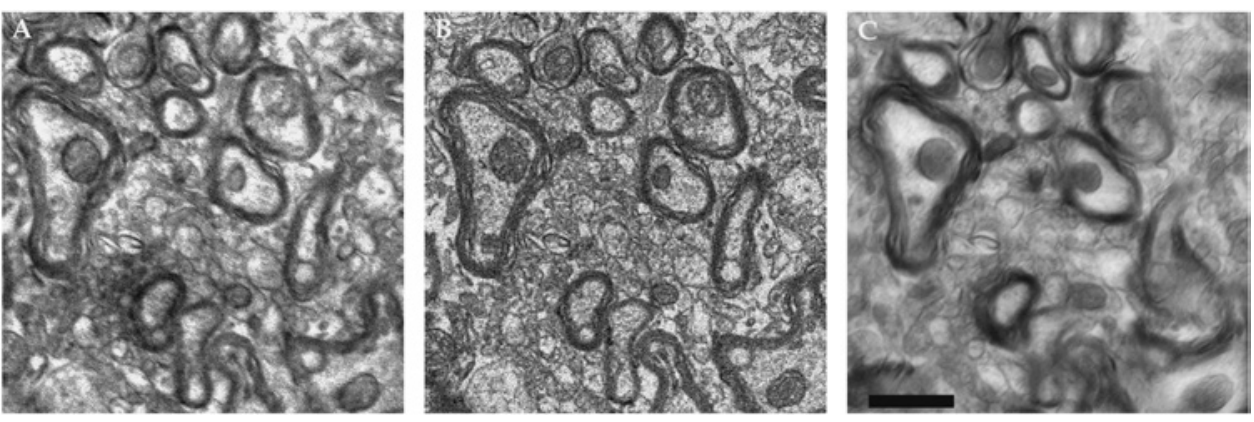

Figure 3: Comparison of images from different detectors. An area of a mouse spinal cord resin-embedded section imaged using three different detectors in the Chipscanner. (A) Everhart-Thornley SE detector. (B) In-lens energyselective backscatter detector (EsB). (C) Angle-selective backscatter electron detector (AsB). Bar = $1 \mu \mathrm{m}$.

the highest signal-to-noise ratio and resolved ultrastructure deep in the sample. All subsequent large-area images were collected using the AsB detector, $10 \mathrm{keV}$ accelerating voltage, $6 \mathrm{nA}$ probe current, and an electron probe size of approximately $6 \mathrm{~nm}$. Next, a standard EBL software routine was employed to calibrate a $100 \mu \mathrm{m}$ FOV, the size of each individual image. All subsequent $100 \mu \mathrm{m}$ FOV images were captured with the FOV corrections.

Producing the mosaic image.

Drift compensation. The Chipscanner's lithographyoriented software has automated drift compensation that can mitigate the beam position drift associated with sample charging and temperature variations. The automated drift compensation routine periodically images a fiducial marker located on or off the sample, and any measured shift in the location is subtracted from subsequent images. The fiducial marker can be any stationary feature in the sample, and of any shape, because the software has image-based cross-correlation algorithms that can measure the shift between two images of the feature. For example, a collection of axon cross sections can make a fiducial marker. Furthermore, the fiducial marker can be anywhere on the sample, or off the sample entirely, because the laser interferometer stage can accurately and repeatedly drive over long distances (multiple FOVs) to the fiducial marker position and back again to continue imaging.

Correction for height differences. The wafer-handling functionality in the Chipscanner, specifically the height-sensing apparatus, lends itself well to the ATUM-SEM approach [3]. In this approach sections of neuronal tissue are laid out across a wafer so that the sections may be non-destructively and repeatedly imaged. This feature is important because the height variation across large areas of sections is typically non-planar.

Specimen preparation. Fixed spinal cords obtained from C57bl6Nmouse with $1.3 \mathrm{Mb}$ deletion of mm.16 (orthologous to the $1.5 \mathrm{Mb}$ "minimal critical deletion region" associated with 22q11.2 DS), also known as LgDel, were cut into $300 \mu \mathrm{m}$ slices, infiltrated with osmium tetroxide and uranyl acetate and embedded in epoxy resin [6,7]. Sections from the entire width of a mouse cervical spinal cord ranging in thickness from $400 \mathrm{~nm}$ to $2 \mu \mathrm{m}$ were cut and placed onto clean silicon wafers mounted on SEM stubs with carbon adhesive tabs.

Imaging conditions. The sections were imaged in a Chipscanner using multiple electron detectors: EverhartThornley secondary electron (SE), in-lens energy-selective backscatter (EsB), and angle-selective backscatter (AsB). Imaging conditions were optimized by comparing images taken using various primary beam energies and electron detectors with the aim of resolving the myelin sheath around the axons. Figure 3 shows a small area of mouse spinal cord containing myelinated axons imaged with three different detectors. In all three cases, the myelin sheaths were resolved. The AsB detector image had
Having optimized the imaging conditions and calibrated the FOV, the Chipscanner was set to automatically capture high-resolution SEM images over a large area of the sample with automatic drift compensation. Each image was $100 \mu \mathrm{m}$ $\times 100 \mu \mathrm{m}$ captured at $10,000 \times 10,000$ pixels corresponding to a $10 \mathrm{~nm} \times 10 \mathrm{~nm}$ pixel size. The large-area image typically has stitching errors well below $40 \mathrm{~nm}$, in the absence of electron beam sample damage effects. To mitigate any residual sample damage effects, stitching errors, or beam position drift, adjacent images are given a small overlap of $100 \mathrm{~nm}$.

\section{Results}

The results of scanning a $2.5 \mathrm{~mm} \times 1.8 \mathrm{~mm}$ area cross section of spinal cord over the course of 24 hours are shown in Figure 4. The sample preparation protocol gives excellent contrast to the cells throughout the spinal cord (Figure 4A). The images were not post-processed by software for stitching error removal. Stitching errors in $X, Y$, and $I$ are barely perceptible, not only for the large-area image mosiac, but also for the magnified images of myelinated axons and motor neurons (Figure 4B1, $4 \mathrm{~B} 2$ yellow arrows, $4 \mathrm{C} 1$ ). Software post-processing was only used to invert the lookup table (LUT), adjust the brightness and contrast, and cropping.

With such a long scanning time, the beam position can drift from sample charging and temperature variation. Automated drift compensation is one way for minimizing errors from the resulting beam position drift. Figure 5 shows how the position of a fiducial marker (a small collection of axon cross sections) changed over 9 hours, where beam position drift corrections were applied roughly every 50 minutes. Before each image was captured, the laser interferometer stage moved distances as high as $2.5 \mathrm{~mm}$ to accurately bring the fiducial marker back into the SEM FOV.

\section{Discussion}

This report shows that the Chipscanner can be used to generate large-area, high-resolution SEM images from spinal cord samples and is therefore a viable method for obtaining relevant detailed information from large biological samples. Large areas of mouse spinal cord were imaged from $2.5 \times 1.8 \mathrm{~mm}$ resin sections placed onto flat silicon wafers. To date, this is the most accurate large-area, high-resolution map of neuronal tissue directly acquired by an SEM instrument. 


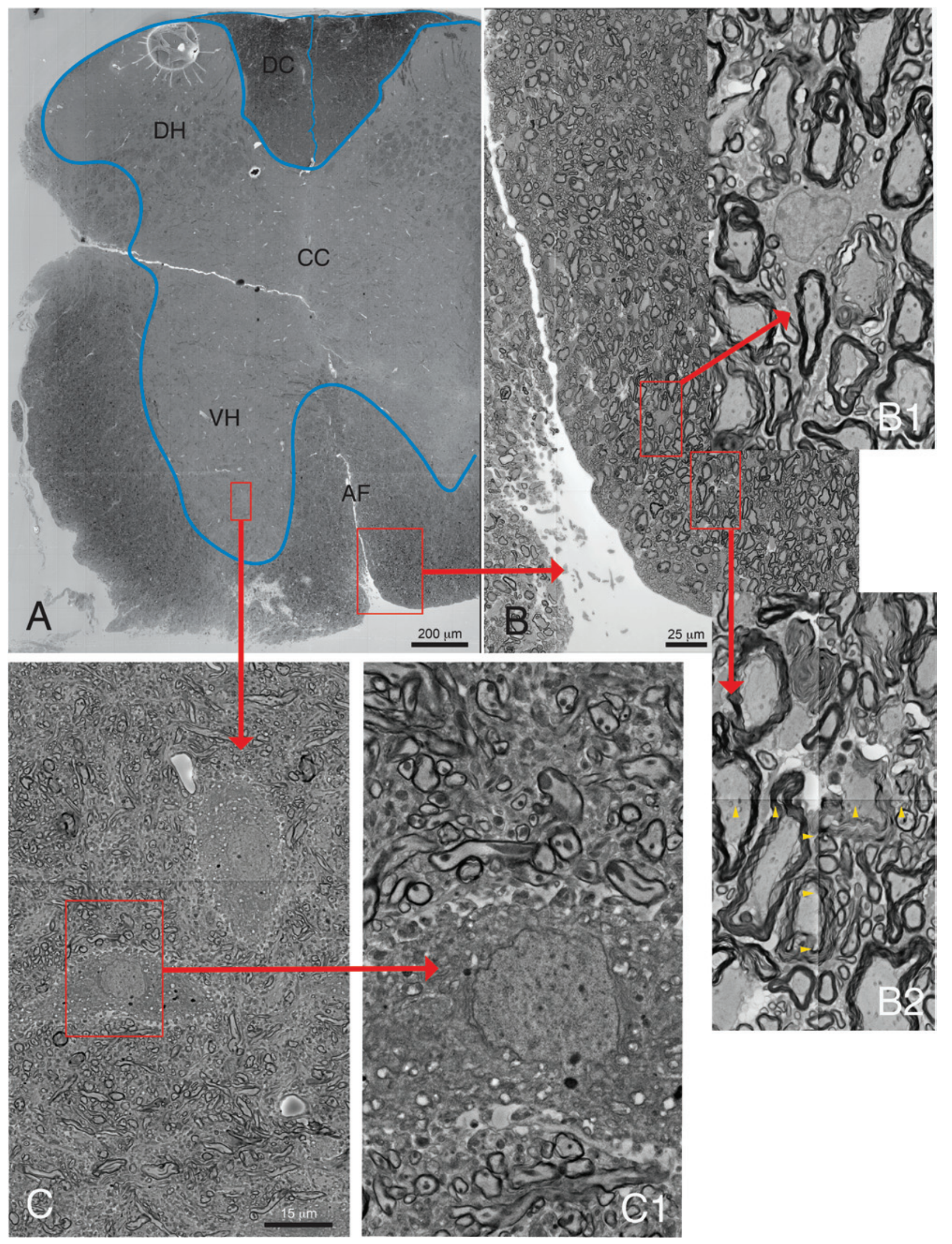

Figure 4: Tiled image (mosaic) of a cross section of LgDel mouse spinal cord. Captured with 450 images, each with a $100 \mu \mathrm{m} \times 100 \mu \mathrm{m}$ FOV. From the large-area mosaic $4 \mathrm{~A}$ high-resolution images can be extracted for further examination. (A) $2.5 \times 1.8 \mathrm{~mm}$ large-area section of spinal cord. Blue outlines delineate the gray matter. Arrows indicate areas in red boxes magnified in adjacent images. (B) $0.24 \times 0.2 \mathrm{~mm}$ section of myelinated axons. (C) $0.12 \times 0.06 \mathrm{~mm}$ section of motor neurons. $\mathrm{DH}=$ dorsal horn, $\mathrm{VH}=\mathrm{ventral}$ horn, $\mathrm{DC}=$ dorsal column, $\mathrm{CC}=$ central canal, $\mathrm{AF}=$ anterior fissura. 

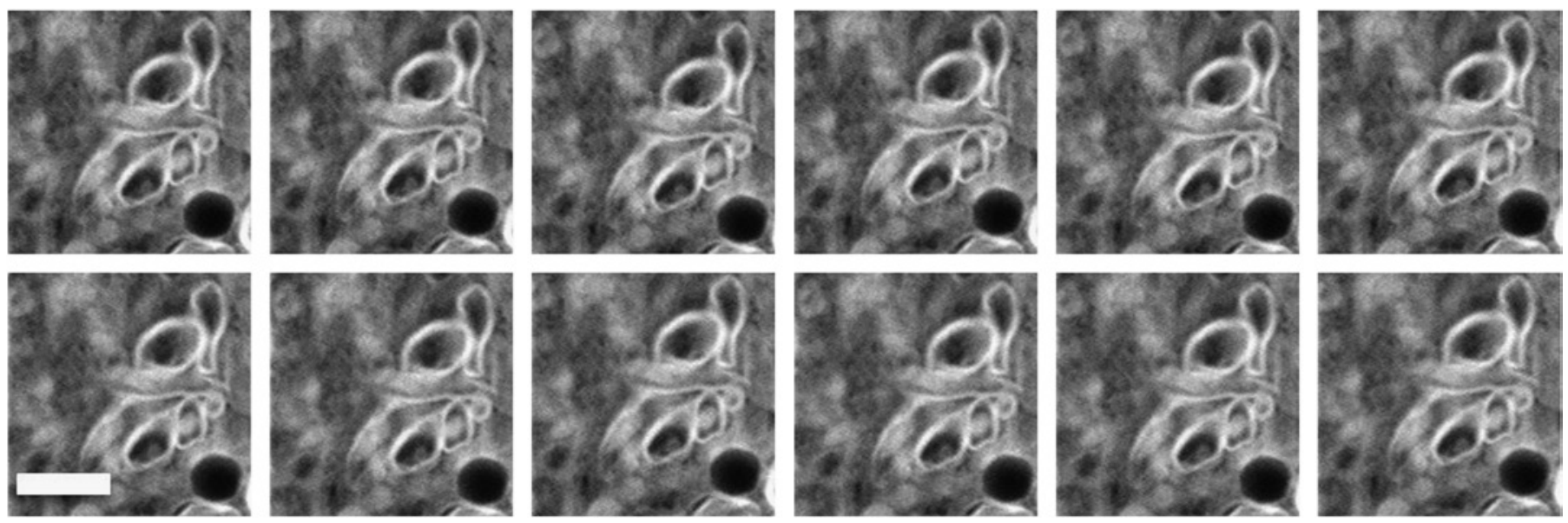

Figure 5: Fiducial feature for automated drift compensation during large-area image capture of mouse spinal cord. The images follow the progression of the fiducial marker (a small collection of axon cross sections) over the span of 9 hours. Bar $=2 \mu \mathrm{m}$.

The Chipscanner can produce large-area mosaic images with stitching errors less than $40 \mathrm{~nm}$ in the absence of electron beam sample damage [4]. This is the first ever application of the Chipscanner for imaging a biological sample. Previous attempts at this type of imaging with other single-beam SEM instruments required, for example, a $4 \mu \mathrm{m}$ overlap [3] due to the lack of a laser interferometer stage and FOV mapping technologies.

Such a large-area map of a sample provides specific location information of a cell or organelle related to its immediate environment, its neighbors, and organization in the tissue, which assists with the assignment of a function to a cell. Large-area imaging can be used to produce accurate counts of objects in a sample or to have a statistically significant number of objects in a sample to measure (that is, axon changes due to treatment, myelin thickness, number of vesicles or mitochondria). For $3 \mathrm{D}$, as has been shown in the reverse engineering of multilevel integrated circuits, the laser interferometer stage and FOV mapping will be powerful assets for accurately stacking images of neuronal tissue.

\section{Conclusion}

The Chipscanner's laser interferometer stage and FOV mapping allow SEM images to be stitched and stacked together to create a high-resolution image encompassing over $2 \mathrm{~mm}$ of specimen. Errors in stitching images together are $40 \mathrm{~nm}$ or less. Such inherently accurate SEM image data minimizes the need for software corrections, which may indeed be impractical or impossible for large data sets for processing limitation reasons. Albeit slower than multiple-beam SEM, such a single-beam SEM offers a wide range of electron detectors and primary beam energies for optimizing imaging conditions.

\section{Acknowledgments}

The authors would like to thank Kevin King for assistance with imaging on the Raith Chipscanner instrument. This work was funded in part by NIH grants P30HD040677 (IDDRC at CNMC) and P01HD083157 (Pathology, Developmental Origins, and Prevention of Pediatric Dysphagia). The authors would like to acknowledge in kind and direct support from Raith during the execution of the work for this article.

\section{References}

[1] AA Wanner et al., Journal Microsc 259 (2015) 137-42.

[2] W Denk et al., PLoS Biology (2004) 1900-09.

[3] KJ Hayworth et al., Frontiers in Neural Circuits 8 (2014) $1-18$.

[4] J Klingfus et al., ISTFA Conference Proceedings (2011) $373-76$.

[5] AL Eberle et al., Microscopy Today 23(2) (2015) 12-18.

[6] T Deerinck et al., Microscopy (2010) 6-8.

[7] K Narayan et al., J Struct Biol 185(3) (2014) 278-84.
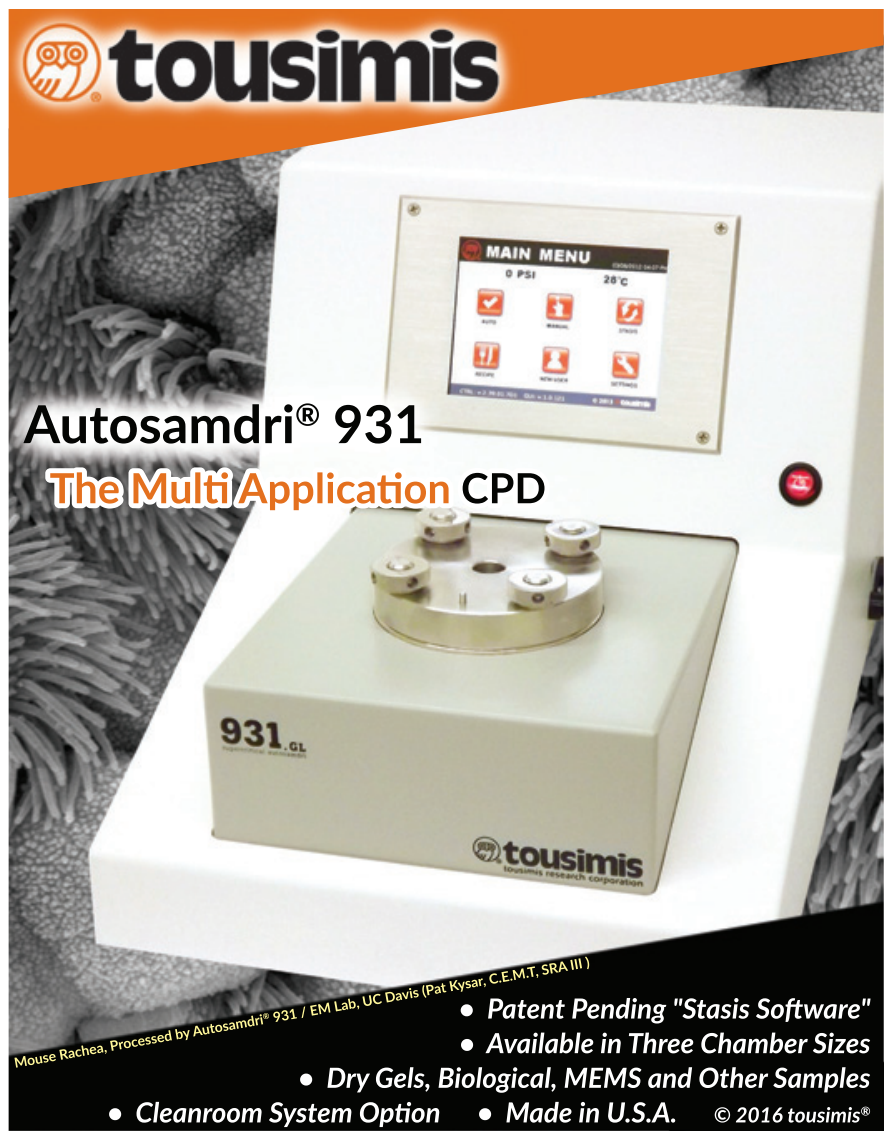